\title{
Are Crackles an Appropriate Outcome Measure for Airway Clearance Therapy?
}

\author{
Alda Marques PhD MSc PT, Anne Bruton PhD MA MCSP, \\ Anna Barney PhD MSc, and Andreia Hall PhD MSc
}

\begin{abstract}
BACKGROUND: There is an urgent need to develop new outcome measures for respiratory therapy, to evaluate its effectiveness. Adventitious sounds generated from the lungs (crackles and wheezes), can now be quantified and characterized objectively with computer technology. To our knowledge, this is the first reported study designed to assess any change in lung crackles before and after a single session of airway clearance therapy. METHODS: Twenty-three stable bronchiectasis patients were recruited from United Kingdom out-patient clinics and treated with a single session of airway clearance therapy, using the active cycle of breathing technique. Sound recordings were made before and after the session at 7 anatomical chest locations. Computerized lung sound analysis was used to measure crackle parameters: 2-cycle deflection width (2CD), and crackle number per breath cycle (nBC). Perceived breathlessness, lung function, and oxygen saturation data were also recorded. RESULTS: Crackle mean $2 \mathrm{CD}$ and mean $\mathrm{nBC}$ increased post intervention. Sixteen participants $(\mathbf{7 0} \%)$ showed a statistically significant difference in mean crackle $2 \mathrm{CD}$ before and after the session at $\geq 1$ chest location. Thirteen $(57 \%)$ participants had a difference between mean crackle 2CD before and after the intervention $>1$ Smallest Real Difference (SRD, mean SRD = $2.23 \mathrm{~ms}$ ) at $\geq 1$ chest location. Differences in mean crackle $\mathrm{nBC}$ before and after the intervention did not exceed the SRD (mean SRD = 32 crackles per breath cycle) in any participant. Perceived breathlessness was significantly reduced post intervention; no significant changes were observed in either lung function or oxygen saturation. CONCLUSIONS: Crackle duration (2CD) was found to change after a single session of airway clearance therapy, and shows promise as a new outcome measure for respiratory therapy interventions. Key words: airway clearance therapy; crackles; lung sounds; outcome measure; respiratory therapy; physical therapy; physiotherapy. [Respir Care 2012; 57(9):1468-1475. () 2012 Daedalus Enterprises]
\end{abstract}

\section{Introduction}

Respiratory therapy is used routinely in clinical practice to manage respiratory problems such as breathlessness, excess lung secretions, reduced lung volumes, and low exercise tolerance. However, little is known about the ef-

\footnotetext{
Dr Marques is affiliated with the School of Health Sciences; and Dr Hall is affiliated with the Department of Mathematics, University of Aveiro, Campus de Santiago, Aveiro, Portugal. Dr Bruton is affiliated with the Department of Respiratory Rehabilitation, Faculty of Health Sciences; and Dr Barney affiliated with the Institute of Sound and Vibration Research, University of Southampton, Highfield, Southampton, United Kingdom.
}

This research was partially supported by Fundação para a Ciência e Tecnologia, Portugal (ref. SFRH/BD/21375/2005). fectiveness of the individual respiratory interventions applied, partly due to the lack of available reliable, valid, sensitive, and specific outcome measures. The outcome measures currently used to assess the effectiveness of respiratory therapy interventions are generally flawed and have been discussed in detail elsewhere. ${ }^{1}$ To our knowledge, this is the first reported study designed to assess any change in lung sounds before and after a single session of airway clearance therapy.

\footnotetext{
Correspondence: Alda Marques PhD MSc PT, School of Health Sciences, University of Aveiro, Campus de Santiago, Edifício III, 3810-193 Aveiro, Portugal. E-mail: amarques@ua.pt.
}

DOI: $10.4187 /$ respcare. 01625 


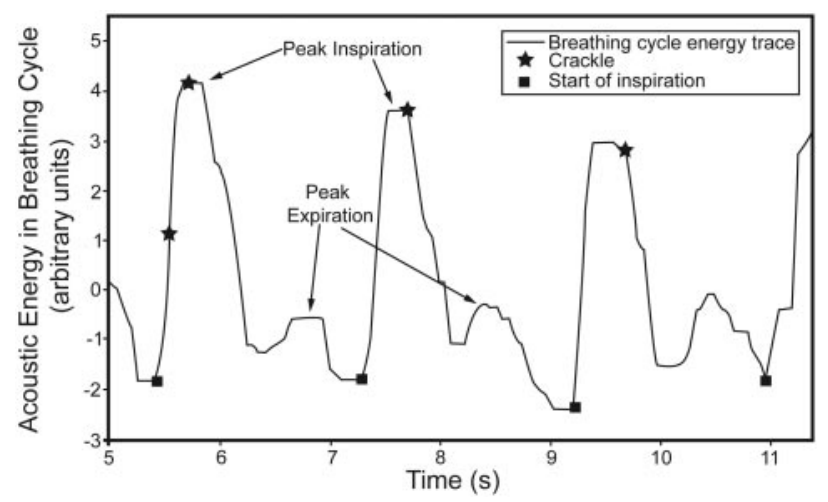

Fig. 1. The solid line show the acoustic energy due to the flow noise during the breathing cycle. Each cycle starts at the point marked with a black square. The first, larger peak in each cycle relates to inspiration, and the second, smaller peak to expiration. The stars are the locations of identified crackles within the breathing cycle. The crackle number per breath cycle $(\mathrm{nBC})$ is the mean number of crackles in each breath cycle in the record. This is calculated by counting the number of crackles in each cycle in the record and averaging over the total number of cycles. In the example shown there are, respectively, 2, 1 , and 1 crackles per sample, so for this extract of the signal $\mathrm{nBC}=1.33$.

Sounds generated from the lungs have the potential to provide useful clinical information, as they relate directly to movement of air, changes within lung tissue and morphology, and secretions. ${ }^{2}$ Research into the acoustic properties of lung sounds has suggested that different parts of the airways produce adventitious lung sounds (such as crackles and wheezes ${ }^{3}$ ) with different characteristics (ie, central airways produce coarser crackles while peripheral airways produce finer $\mathrm{crackles}^{4-6}$ ). The technique of computer aided lung sounds analysis (CALSA), applying expanded time and spectral analysis techniques to crackle and wheeze parameters, ${ }^{4}$ has been used to detect and characterize the severity of lung disease, ${ }^{5,6}$ but it has yet to be evaluated as an outcome measure for respiratory therapy. The use of CALSA to characterize crackles over short periods has been shown to provide an objective and reliable measure in a clinical setting. ${ }^{7}$ However, before crackle analysis can be recommended as an outcome measure for respiratory clinical practice, it is necessary to demonstrate that crackle parameters change after an intervention.

Respiratory clinicians describe crackles as many or few, early or late, coarse or fine. Using CALSA, crackles can be objectively characterized based on their number per breath cycle (nBC, Fig. 1) or their duration. The 2-cycle deflection width (2CD) is the duration of the first 2 cycles of a crackle, in milliseconds (Fig. 2). Crackle 2CD has been shown to be a more stable and reliable measure than another parameter measuring crackle duration, the initial deflection width. ${ }^{7}$

\section{QUICK LOOK}

\section{Current knowledge}

Breath sounds determined using traditional auscultation are frequently used to evaluate the success of airway clearance maneuvers. The subjective nature of breath sounds and variations related to technique render these evaluations suboptimal.

\section{What this paper contributes to our knowledge}

Computer aided lung sound analysis represents an objective measure for monitoring respiratory therapy, including airway clearance maneuvers. The measurement of the 2-cycle detection width and the duration of crackles (in milliseconds) appear to be stable and reliable measurements.

The aim of this study was to explore whether crackle parameters are appropriate for use as an outcome measure in respiratory practice, with 2 specific objectives:

- To investigate whether crackle $2 \mathrm{CD}$ or crackle $\mathrm{nBC}$ is affected by the application of a single session of an airway clearance intervention.

- To assess the expected effect size for crackle 2CD and crackle $\mathrm{nBC}$ by calculating the smallest real difference (SRD). ${ }^{8}$

\section{Methods}

This study was conducted in the Faculty of Health Sciences, University of Southampton, Highfield, Southampton, United Kingdom.

\section{Design}

A single group, repeated measures design was used, in which sets of lung sound recordings were made from the same participants twice on the same day, 3 before and 3 after a single airway clearance intervention.

\section{Participants, Centers}

The study received full approval from the Southampton and South West Hampshire Research Ethics Committee (A). Twenty-three participants were recruited for this study via bronchiectasis out-patient clinics held at Queen Alexandra Hospital, Portsmouth, on the south coast of the United Kingdom. Participants were eligible if they were able to give and sign informed consent; had a documented medical diagnosis of bronchiectasis, made by a consultant re- 


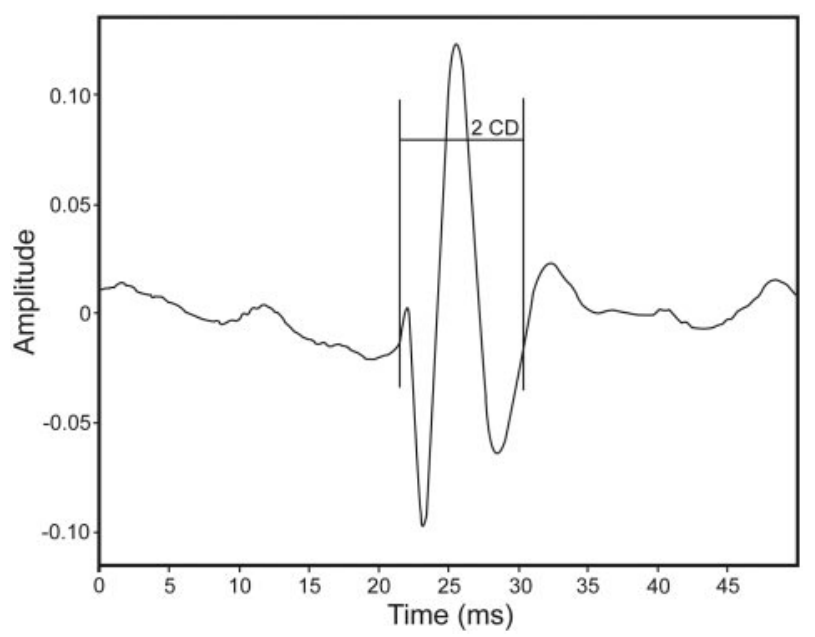

Fig. 2. Representation of crackle 2-cycle deflection width (2CD).

spiratory physician; were $\geq 18$ years of age; and had been clinically stable for one month prior to the study (defined as no hospital admissions, no exacerbations/infections, and no change in prescribed medication). Patients were excluded from the study if they had co-existing lung pathologies.

\section{Sample Size}

A convenience sample was recruited since no prior information on expected sample variance in crackle parameters was available to permit a power calculation to determine sample size.

\section{Intervention}

A standard airway clearance technique (active cycle of breathing technique) was performed with each participant, by the same experienced respiratory physiotherapist, for a single treatment session in an out-patient setting. Active cycle of breathing technique is the most frequently used airway clearance technique in the United Kingdom. ${ }^{9} \mathrm{Cy}-$ cles were repeated until the participant felt his or her chest to be clear. On average, each session lasted $24 \mathrm{~min}$, with a range between 15 and $30 \mathrm{~min}$. Typically, airway clearance treatment sessions last from $20-30 \mathrm{~min} .{ }^{10}$

\section{Outcome Measures}

The modified Borg Scale ${ }^{11}$ was used to collect perceived breathlessness data. $\mathrm{FEV}_{1}$, FVC, and peak expiratory flow (PEF) were recorded using a spirometer (Microlab 3500, Micro Medical/Carefusion, San Diego, California), which was calibrated daily; and oxygen saturation data were collected with a pulse oximeter (Pneupac, Sims, Luton, United Kingdom). Lung sound recordings were performed with an electronic stethoscope (Master Elite, Welch Allyn, Skaneateles Falls, New York). The output from the stethoscope was connected via an integral amplifier to the sound card of a laptop with customized software suitable for data acquisition, written in Matlab 7.1 (Mathworks, Natick, Massachusetts). Lung sounds were recorded with a sampling frequency of $44.1 \mathrm{kHz}$ and a quantization of 16 bits for all recordings.

Demographic and basic anthropometric data (height and weight) were recorded first. Breathlessness and oxygen saturation levels were then registered. Baseline lung sound recordings were performed with the electronic stethoscope held by hand over each location (previously marked in pen on the patient's chest to ensure that the stethoscope was placed on the same location) for 25 seconds per recording. Three recordings per location were made at baseline to provide data for a reliability study published elsewhere. ${ }^{7}$ For this before-after study, one of the 3 baseline recordings was selected at random for analysis. Lung sound recordings followed the guidelines for short-term acquisition from the Computerized Respiratory Sound Analysis (CORSA) project group. ${ }^{12}$ Although the trachea is one of the 7 recording sites recommended in the CORSA guidelines, Gavriely and Cugell ${ }^{13}$ have reported that lung sounds from the trachea are differently filtered than those from other sites. In addition, clinicians rarely use this site for assessing crackles. It was therefore decided not to include tracheal data for this study. Spirometry was performed in accordance with published guidelines, ${ }^{14}$ after the lung sound recordings. After the intervention, lung sound recordings, breathlessness, oxygen saturation, and lung function tests were repeated, using the same procedure as at baseline. Lung function tests were performed after the lung sounds recordings (approximately $15 \mathrm{~min}$ after the intervention), because forced expiratory maneuvers could affect lung sounds.

\section{Data Analysis}

All tests for statistical significance were at the $95 \%$ level.

\section{Analysis of Demographic and Anthropometric Data}

Body mass index (BMI), in $\mathrm{kg} / \mathrm{m}^{2}$, was calculated using the formula BMI $=$ weight $/(\text { height })^{2}$. Descriptive statistics were used to summarize the sample.

\section{Analysis of Breathlessness, Oxygen Saturation and Lung Function}

Analysis was based on data collected at baseline and immediately post intervention. The predicted values used for lung function were based on the European Respiratory 
Society reference values, ${ }^{15}$ and the highest of the 3 spirometric measures performed at baseline and post intervention was used for analysis. The comparisons (at baseline and post intervention) for the lung function and oxygen saturation variables were made using a paired sample $t$ test. Breathlessness comparisons were made using the nonparametric Wilcoxon signed rank test. Any statistically significant results were explored further by examining for changes exceeding the published minimally clinically important difference. ${ }^{16}$

\section{Lung Sound Analysis}

All sound files were processed using algorithms written in Matlab. Crackles were detected based on an algorithm developed by Vannuccini et al, ${ }^{17}$ which has been validated for sensitivity (84\%) and specificity (89\%) against clinical data. The reasons for this choice have been discussed elsewhere. ${ }^{7}$

\section{Breathing Cycle Detection}

A method analogous to that reported by Que et al ${ }^{18}$ was used to detect the breath cycles automatically. However, since a fully automated breath cycle detection process was not found to be reliable in the presence of substantial numbers of crackles, manual adjustment of the detection thresholds was permitted. Threshold adjustment was based on visual inspection of the plotted signal, and aural extraction of the breath cycle phases by a physiotherapist familiar with auscultation techniques.

\section{Crackle Parameter Analysis}

A database was created for the data (at both baseline and post intervention) at each chest location, per participant, for crackle $2 \mathrm{CD}$ and crackle $\mathrm{nBC}$. Mean $\mathrm{nBC}$ was calculated by averaging $\mathrm{nBC}$ across all complete breath cycles in the recorded signal for a given participant and chest location. Mean 2CD was calculated by averaging across all crackles in the recorded signal for a given participant and chest location. For each participant and each chest location the difference in mean $\mathrm{nBC}$ and in mean $2 \mathrm{CD}$ pre and post intervention was compared by a paired $t$ test.

The group mean $2 \mathrm{CD}$ and group mean $\mathrm{nBC}$ detected pre intervention in each of the 6 chest locations were compared to the corresponding post intervention group means using a 2-way analysis of variance, where the participant was considered a random factor and the time (pre/post intervention) was a fixed, repeated measures factor.

For crackle parameters, the minimally clinically important difference has yet to be determined, so the SRD was chosen to explore the size of any effect. The SRD repre- sents the smallest change that can be interpreted as a real difference in excess of measurement "noise" 8 and is estimated by SRD $=1.96 \sqrt{ } 2$ (SEM). The standard error of measurement (SEM) was obtained from the baseline data by calculating the square root of the within participant mean square values from the analysis of variance table generated by comparing the mean dependent variable $(2 \mathrm{CD}$, $\mathrm{nBC}$ for a given location across all participants) in 2 sets of pre intervention data selected at random for the 3 data sets recorded for each subject. The difference between the pre and post intervention means of the crackle $2 \mathrm{CD}$ for each chest location for each participant was obtained by subtracting the mean $2 \mathrm{CD}$ after treatment from the mean $2 \mathrm{CD}$ before treatment. The corresponding calculation was also made for the mean $\mathrm{nBC}$. Participants with a difference at any chest location greater than \pm 1 SRD were identified.

Finally, any systematic bias in the difference of the pre and post intervention means was sought using Bland-Altman plots. ${ }^{18}$ These plots provide a visual representation of agreement, with easy identification of systematic bias, outliers, and any relationship between the variance in measures with the size of the mean.

\section{Results}

Twenty-three participants (14 female, 61\%) of $61.2 \pm 9.6$ years old (age range $25-73$ years old), were recruited. The average BMI of the sample $\left(28.3 \pm 5.6 \mathrm{~kg} / \mathrm{m}^{2}\right)$ was higher than deemed "normal." The majority of crackles were coarse $(2 \mathrm{CD}>10 \mathrm{~ms})$ and occurred early in the breathing cycle, which is compatible with previously published data from patients with bronchiectasis. ${ }^{19}$

\section{Breathlessness, Oxygen Saturation, and Lung Function}

The group mean breathlessness score was statistically significantly lower after the airway clearance intervention $(P=.02)$. Twelve out of the 23 participants $(52 \%)$ perceived themselves to be less breathless, in comparison to their baseline perception, and in 10 of these the difference was equal to or exceeded the published minimally clinically important difference for the modified Borg score (1 unit). ${ }^{16}$ Post hoc Cohen's $d$ was calculated as 0.4 , with an effect size $r$ of 0.2 , which indicates a small to medium effect. ${ }^{20}$

The group means at baseline and post airway clearance intervention for the oxygen saturation levels and each lung function variable were similar, and no statistically significant differences were found (Table 1 and Table 2). 
Table 1. Descriptive Statistics for Breathlessness Data (Modified Borg Scale) Before and After Intervention With Wilcoxon Signed Rank Test Results $(n=23)$

\begin{tabular}{lccccc}
\hline \hline Timing & $\begin{array}{c}\text { Median } \\
\text { (IQR) }\end{array}$ & $\begin{array}{c}\text { Min-Max, } \\
\text { no. }\end{array}$ & $\begin{array}{c}\text { Negative } \\
\text { Ranks, } \\
\text { no.* }\end{array}$ & $\begin{array}{c}\text { Positive } \\
\text { Ranks, } \\
\text { no. } \dagger\end{array}$ & $\begin{array}{c}\text { Tie, } \\
\text { no. } \ddagger\end{array}$
\end{tabular}

Table 2. Descriptive Statistics for Oxygen Saturation and Spirometry Data Before and After Intervention With Paired $t$ Test Results and 95\% Confidence Intervals for the Difference Between the Means $(n=23)$

\begin{tabular}{llrrr}
\hline \hline \multicolumn{1}{c}{ Variable } & Timing & Mean $\pm \mathrm{SD}$ & $P$ & $\begin{array}{c}\text { 95\% CI of the } \\
\text { Difference }\end{array}$ \\
\hline $\mathrm{S}_{\mathrm{PO}_{2}, \%}$ & Pre & $95 \pm 2.5$ & .07 & -0.04 to 1.1 \\
$\mathrm{FVC}, \mathrm{L}$ & Post & $94 \pm 2.1$ & & \\
& Pre & $2.6 \pm .77$ & .16 & -0.03 to 0.15 \\
$\mathrm{FEV}_{1}, \mathrm{~L}$ & Post & $2.5 \pm .79$ & & \\
& Pre & $1.4 \pm .62$ & .08 & -0.01 to 0.05 \\
$\mathrm{FEV}_{1} / \mathrm{FVC}$ & Post & $1.4 \pm .63$ & & \\
& Pre & $54 \pm 17$ & .30 & -5.4 to 1.7 \\
$\mathrm{FEV}_{1}, \%$ predicted & Post & $56 \pm 18$ & & \\
& Pre & $60 \pm 29$ & .08 & -0.13 to 2.2 \\
$\mathrm{FVC}, \%$ predicted & Post & $59 \pm 29$ & & \\
& Pre & $80 \pm 20$ & .20 & -1.1 to 4.9 \\
PEF, L/min & Post & $78 \pm 21$ & & \\
& Pre & $240 \pm 106$ & .18 & -3.1 to 16 \\
& Post & $234 \pm 99$ & & \\
\hline PEF = peak expiratory flow & & & & \\
\hline
\end{tabular}

\section{Crackle 2CD and nBC}

Pre intervention an average across all chest locations and all participants of $4.14 \pm 2.31$ crackles per BC was detected, with a $2 \mathrm{CD}$ of $11.8 \pm 1.50 \mathrm{~ms}$. Post intervention an average across all chest locations and all participants of $4.18 \pm 2.25$ crackles per $\mathrm{BC}$ was detected, with a $2 \mathrm{CD}$ of $11.9 \pm 1.54 \mathrm{~ms}$. Neither the group mean $2 \mathrm{CD}$ nor the group mean $\mathrm{nBC}$ showed statistically significant change from pre to post intervention at any chest location.

The results above relate to average changes across the group; however, in this study our main interest was in exploring any changes that occurred within individuals from before to after the intervention. We therefore quantified these individual changes. When the individual data were analyzed, there were statistically significant changes
Table 3. Smallest Real Difference Values Obtained From the Analysis of Mean Crackle 2CD and Mean nBC at Baseline, and the Number of Participants With a Difference Before and After Treatment $>1$ SRD, at Each of the 6 Chest Locations

\begin{tabular}{lcccc}
\hline \hline & $\begin{array}{c}\text { Baseline } \\
\text { Crackle } \\
\text { 2CD/BC SRD } \\
\text { (ms) }\end{array}$ & $\begin{array}{c}\text { No. With } \\
\text { a Change } \\
\text { > 1 Crackle } \\
\text { 2CD/BC SRD } \\
\text { (no.) }\end{array}$ & $\begin{array}{c}\text { Baseline } \\
\text { Crackle } \\
\text { nBC SRD } \\
\text { (no.) }\end{array}$ & $\begin{array}{c}\text { No. With a } \\
\text { Change } \\
\text { 1 Crackle } \\
\text { nBC SRD } \\
\text { (no.) }\end{array}$ \\
\hline Anterior right & 3.1 & 2 & 13 & 0 \\
Anterior left & 2.0 & 3 & 16 & 0 \\
Lateral right & 2.2 & 3 & 51 & 0 \\
Lateral left & 1.8 & 7 & 41 & 0 \\
Posterior right & 2.6 & 0 & 37 & 0 \\
Posterior left & 2.0 & 5 & 34 & 0 \\
$\begin{array}{l}\text { 2CD }=2 \text {-cycle deflection width } \\
\text { nBC }=\text { crackle number per breath cycle }\end{array}$ & & & \\
SRD $=$ smallest real difference & & & \\
\end{tabular}

in mean $2 \mathrm{CD}$ within 16 participants (70\%). The direction of change was inconsistent, although there were twice as many cases of significant mean $2 \mathrm{CD}$ increase (coarser crackles) as there were cases of significant mean $2 \mathrm{CD}$ decrease. The significant changes recorded at the posterior right location were uniformly in the direction of an increase in mean $2 \mathrm{CD}$ after the airway clearance intervention (this was seen in 5 participants). Seven participants $(30 \%)$ showed no significant change in mean $2 \mathrm{CD}$ at any location. When mean $\mathrm{nBC}$ data were analyzed in the same way, there were statistically significant changes in mean $\mathrm{nBC}$ within 10 participants (43\%). The direction of change was inconsistent, but there were twice as many cases of significant mean $\mathrm{nBC}$ decrease (fewer crackles) as there were cases of significant mean $\mathrm{nBC}$ increase. Nine of the 19 examples (47\%) of significant changes were seen in the posterior locations.

\section{Smallest Real Difference}

Table 3 shows that the baseline SRD values varied across chest locations for the mean $2 \mathrm{CD}$ and mean $\mathrm{nBC}$. The same table also shows the number of participants who presented a change $>1 \mathrm{SRD}$ in mean $2 \mathrm{CD}$ or $\mathrm{nBC}$ from pre to post intervention. At the $95 \%$ level it would be expected that from a sample of 23 people, approximately one participant would show such change by chance. It is evident that mean $2 \mathrm{CD}$ showed a change $>1 \mathrm{SRD}$ at all chest locations (except the posterior right) in more participants than would be anticipated by chance at the $95 \%$ level, but mean $\mathrm{nBC}$ did not. Of the 23 participants, 13 $(57 \%)$ had changes in mean $2 \mathrm{CD}>1 \mathrm{SRD}$ at a minimum of one location. The lateral locations recorded the majority 


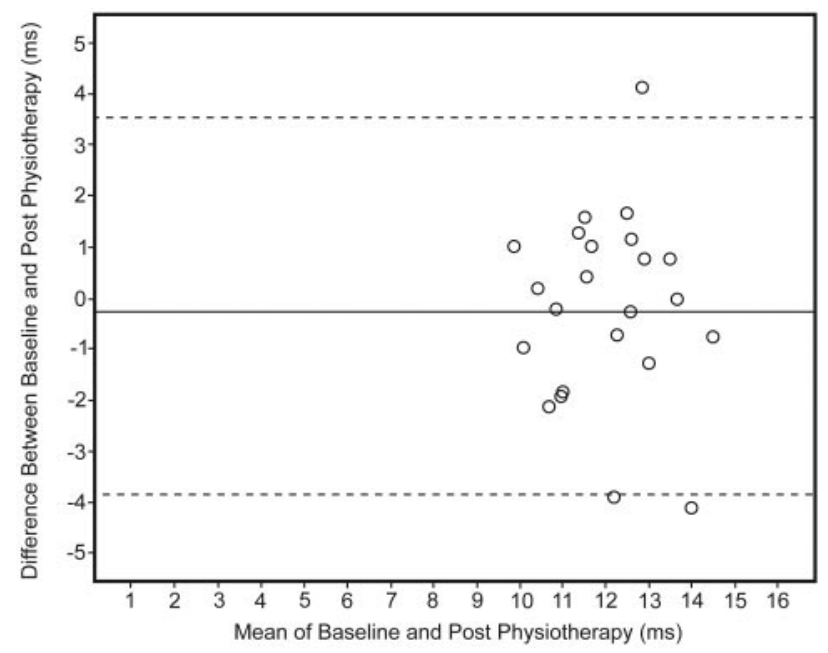

Fig. 3. Bland-Altman plot showing $95 \%$ limits of agreement for the crackle 2-cycle deflection width $(n=23)$ at the anterior right of the chest, using data at baseline and post airway clearance intervention.

of changes $>1 \mathrm{SRD}$, with the left lateral location recording changes $>1$ SRD in 7 participants (30\%). None of the participants had a change in mean $\mathrm{nBC}>1 \mathrm{SRD}$ at any location.

\section{Bland-Altman Plots}

Bland-Altman plots revealed the existence of some systematic bias between pre and post intervention for mean $2 \mathrm{CD}$, but not for mean $\mathrm{nBC}$. Figure 3 shows a typical example using the mean $2 \mathrm{CD}$ data.

\section{Discussion}

This research has shown that in stable bronchiectasis no statistically significant change at the $95 \%$ level was seen in group means for crackle $2 \mathrm{CD}$ and $\mathrm{nBC}$ from before to after a single session of airway clearance. However, individual participants frequently had statistically significant changes in one or both parameters. For each anatomical chest location, more participants showed a change in mean $2 \mathrm{CD}>1 \mathrm{SRD}$ (derived from the baseline data) than would be expected through chance alone, although the direction of this change was not consistent across participants. The left lateral recording location produced the largest number of changes in $2 \mathrm{CD}>1$ SRD. No corresponding pattern was found relating to the mean $\mathrm{nBC}$.

The intervention was standardized by ensuring that the same physiotherapist performed all maneuvers and used a standard airway clearance technique (active cycle of breathing technique). However, in accordance with normal clinical practice, the physiotherapist had some flexibility in terms of the duration of the single treatment session, continuing until the participants felt their chest to be "clear."

There is no gold standard to determine effectiveness of airway clearance, so the effectiveness of the intervention provided in this study is uncertain. It is possible that the observed changes in each individual for the mean crackle $2 \mathrm{CD}$ and mean crackle $\mathrm{nBC}$ occurred by chance, as this was an exploratory study and no control group was included. However, this is unlikely, as statistically significant changes were observed in 16/23 patients for the mean crackle $2 \mathrm{CD}$, and in $10 / 23$ patients for the mean crackle $\mathrm{nBC}$. The fact that the direction of change was inconsistent and that in some patients no change was observed also supports this argument, as the session of airway clearance might not have cleared the patient's chest or might not have been sufficient to produce a measurable effect in some patients.

In this study air flow was not standardized, which might have affected the results. However, of all the methods to measure flow, the pneumotachograph has been considered the gold standard, ${ }^{21}$ but the patient has to breathe using a mouthpiece or a face mask. This causes patient discomfort, is not practical, and changes the breathing pattern and therefore is rarely used clinically.22,23 Furthermore, gathering breathing cycle data from non-acoustic means (eg, from pneumotachographs) may be difficult or impossible in some populations and pathologies. These aspects have also been acknowledged by other researchers. ${ }^{24}$ Breathing cycle detection without air flow measurements has been successfully achieved. ${ }^{24-26}$ However, these researchers used 6 simultaneous microphones attached to the trachea and chest, and the data were recorded in a respiratory acoustics laboratory and on healthy subjects. An acoustical approach to respiratory phase detection is attractive, because it is objective, noninvasive, relatively inexpensive, and convenient to use ${ }^{24}$ in a clinical setting. Our use of it in this study was intended to explore an outcome measure to be used clinically.

In the absence of a gold standard to define the magnitude of minimally clinically important changes, the SRD provides evidence of a real change that is not attributable to "error" or "noise." ${ }^{8}$ Changes smaller than the SRD should be interpreted as measurement error, while changes larger than the SRD can be interpreted as a real change in values. In this study, differences in mean $2 \mathrm{CD}$ were greater than the SRD in several participants at each chest location except the posterior right. This supports the hypothesis that crackle mean $2 \mathrm{CD}$ is responsive to change. No participants demonstrated a difference $>1$ SRD for mean $\mathrm{nBC}$. This suggests that mean $\mathrm{nBC}$ is a stable parameter that is not affected by airway clearance techniques, or that it is not responsive to change. Vyshedskiy et $\mathrm{al}^{27}$ have recently reported on the stability of crackle rate within single examination sessions, despite intervening maximal breathing 
maneuvers. Recording for 25 second periods provided sufficient data for our crackle analysis. However, there was considerable variability in the interaction effect between subject and data sample for the number of crackles per breath cycle, which resulted in a very high variance when calculating the $\mathrm{nBC}$ SRD figures per location. It is possible, therefore, that $\mathrm{nBC}$ might be responsive to change if recordings were taken for longer periods, but a requirement for longer recording periods would be less practical in clinical settings.

Bland-Altman plots revealed the presence of some systematic bias toward an increase in mean crackle $2 \mathrm{CD}$ ("coarser" crackles) after intervention in recordings made at anterior and lateral chest regions. Crackles heard here post intervention may therefore originate from more central airways, since smaller airways are believed to produce fine, late inspiratory crackles, whereas larger airways tend to produce coarser, earlier crackles. ${ }^{28} \mathrm{~A}$ movement of crackles to more central airways may arise as a result of forced expiratory maneuvers moving secretions and allowing more air to pass. The association between crackle classification (fine vs coarse) and the location in the airways of their generation has been previously investigated by Fredberg and Holford, ${ }^{29}$ and has been put on a firmer basis by Majumdar et al. ${ }^{30}$

Although CALSA has not previously been used as an outcome measure for therapeutic interventions, it has been reported that it can detect changes during the course of pharmacologically induced airway obstruction, ${ }^{31}$ during forced expiratory maneuvers, ${ }^{32}$ and during pathological processes such as pneumonia. ${ }^{6,33}$ Baughman and Loudon ${ }^{5}$ recorded lung sounds in asthma patients overnight and were able to detect degrees of obstruction severity not revealed by any other measure. CALSA may therefore provide a more sensitive measure to detect alterations in airway geometry than conventional outcome measures.

Devices that record and analyze wheezes are already in clinical use (eg, Wheezometer, Karmel Sonix, Haifa, Israel), but the technology for instant crackle analysis clinically at the bedside is not yet commercially available. The aim is to produce a simple, reliable device that can record and analyze sounds at the bedside to provide clinicians with immediate information about a patient's lung health. This would provide objective information not only in terms of whether an individual was improving, stable, or deteriorating in relation to previous recordings, or in response to an intervention, but also in relation to normative data (similar to comparisons for lung function data). Before this can be achieved, there is further work to be done in terms of validation studies (eg, against expert opinion and objective imaging data), and further work on the signal processing techniques to provide real-time automated analysis. The main cost at present is in relation to the research and development required. Once a commercial system is available, further health economic evaluations will be required to determine the cost-benefit or otherwise of implementing such technology in clinical practice.

\section{Conclusions}

Respiratory therapy urgently needs robust outcome measures to monitor and evaluate effectiveness and to produce an evidence base. Lung sounds contain a wealth of clinically useful information that has the potential to be used to evaluate treatment. To our knowledge, this study is the first to estimate change in the number and duration of specific adventitious lung sounds (ie, crackles) after a therapeutic intervention. Mean $\mathrm{nBC}$ did not change, but mean $2 \mathrm{CD}$ did change within individuals. We have previously reported that the $2 \mathrm{CD}$ is a stable and reliable measure over short periods of time. ${ }^{7}$ The findings of this research therefore provide further indication that $2 \mathrm{CD}$ has potential for application as a new individual outcome measure in respiratory clinical practice.

\section{ACKNOWLEDGMENTS}

The authors would like to thank Prof Paul White, University of Southampton, Highfield, Southampton, United Kingdom, for useful discussions regarding this study. We are also very grateful to all the participants for their involvement and to the staff of the Respiratory Center at Queen Alexandra Hospital, Portsmouth, United Kingdom.

\section{REFERENCES}

1. Marques A, Bruton A, Barney A. Clinically useful outcome measures for physiotherapy airway clearance techniques: a review. Phys Ther Rev 2006;11(4):299-307.

2. Kiyokawa H, Pasterkamp H. Volume-dependent variations of regional lung sound, amplitude and phase. J Appl Physiol 2002;93(3): 1030-1038.

3. Piirila P, Sovijarvi ARA. Crackles: recording, analysis and clinical significance. Eur Respir J 1995;8(12):2139-2148.

4. Charbonneau G, Ademovic E, Cheetham BMG, Malmberg LP, Vanderschoot J, Sovijarvi ARA. Basic techniques for respiratory sound analysis. Eur Respir Rev 2000;10(77):625-635.

5. Baughman RP, Loudon RG. Lung sound analysis for continuous evaluation of airflow obstruction in asthma. Chest 1985;88(3):364368.

6. Piirila P. Changes in crackle characteristics during the clinical course of pneumonia. Chest 1992;102(1):176-183.

7. Marques A, Bruton A, Barney A. Reliability of lung crackle characteristics in cystic fibrosis and bronchiectasis patients in a clinical setting. Physiol Meas 2009;30(9):903-912.

8. Beckerman H, Roebroeck ME, Lankhorst GJ, Becher JG, Bezemer PD, Verbeek AL. Smallest real difference, a link between reproducibility and responsiveness. Qual Life Res 2001;10(7):571-578.

9. O'Neill B, Bradley J, McArdle N, MacMahon J. The current physiotherapy management of patients with bronchiectasis: a UK survey. Int J Clin Pract 2002;56(1):34-35.

10. Prasad SA, Main E. Finding evidence to support airway clearance techniques in cystic fibrosis. Disabil Rehabil 1998;20(6-7):235-246.

11. Borg GA. Psychological basis of perceived exertion. Med Sci Sport Exerc 1982;14(5):377-381. 
12. Sovijarvi ARA, Vanderschoot J, Earis JE. Computerized respiratory sound analysis (CORSA): recommended standards for terms and techniques. ERS Task force report. Eur Respir Rev 2000;10(77):595649.

13. Gavriely N, Cugell DW. Airflow effects on amplitude and spectral content of normal breath sounds. J Appl Physiol 1996;80(1):5-13.

14. Miller MR, Hankinson J, Brusasco V, Burgos F, Casaburi R, Coates A, et al. Standardisation of spirometry. Eur Respir J 2005;26(2):319338.

15. Quanjer PH, Tammeling GJ, Cotes JE, Pederson OF, Peslin R, Yernault JC. Lung volumes and forced expiratory flows. Report working party standardization of lung function tests, European Community for Steel and Coal. Official Statement of the European Respiratory Society. Eur Respir J 1993;6(Suppl 16):5-40.

16. Ries AL. Minimally clinically important difference for the UCSD Shortness of Breath Questionnaire, Borg Scale and Visual Analogue Scale COPD 2005;2(1):105-110.

17. Vannuccini L, Rossi M, Pasquali G. A new method to detect crackles in respiratory sounds. Technol Health Care 1998;6(1):75-79.

18. Que CL, Kolmaga C, Durand LG, Kelly SM, Macklem PT. Phonospirometry for noninvasive measurement of ventilation: methodology and preliminary results. J Appl Physiol 2002;93(4):1515-1526.

19. Piirila P, Sovijarvi ARA, Kaisla T, Rajala H-M, Katila T. Crackles in patients with fibrosing alveolitis, bronchiectasis, COPD, and heart failure Chest 1991;99(5):1076-1083.

20. Cohen J. Statistical power analysis for the behavioral sciences. Lawrence Earlbaum Associates: Hillsdale, NJ; 1988.

21. Brouwer AFJ, Roorda RJ, Brand PL. Comparison between peak expiratory flow and $\mathrm{FEV}_{1}$ measurements on a home spirometer and on a pneumotachograph in children with asthma. Pediatr Pulm 2007; 42(9):813-818.

22. Akre H, Borgersen AK, Mair IWS, Skatvedt O. Tracing air flow and diagnosing hypopnoeas in normal subjects. Physiol Meas 2000;21(2): 221-227.

23. Manczur T, Greenough A, Hooper R, Allen K, Latham S, Price JF, et al. Tidal breathing parameters in young children: comparison of measurements by respiratory inductance, plethysmography to a facemask, pneumotachograph system. Pediatr Pulm 1999;28(6):436-441.

24. Chuah JS, Moussavi ZK. Automated respiratory phase detection by acoustical means. Syst Cybernet Informatics (SCI) Conf 2000;228231.

25. Moussavi ZK, Leopando MT, Pasterkamp H, Rempel G. Computerized acoustical respiratory phase detection without airflow measurement. Med Biol Eng Comput 2000;38(2):198-203.

26. Moussavi ZK, Leopando MT, Rempel GR. Automated detection of respiratory phases by acoustical means. Proc 20th Internat Conf IEEE. Eng Med Biol Soc 1998;20(1):21-24.

27. Vyshedskiy A, Ishikawa S, Murphy R. Crackle pitch and rate do not vary significantly during a single examining session in patients with pneumonia, congestive heart failure and interstitial pulmonary fibrosis. Respir Care 2011;56(6):806-817.

28. Sovijarvi ARA, Malmberg LP, Charbonneau G, Vanderschoot J, Dalmasso F, Sacco C, et al. Characteristics of breath sounds and adventitious respiratory sounds. Eur Respir Rev 2000;10(77):591596

29. Fredberg JJ, Holford SK. Discrete lung sounds: crackles (rales) as stress-relaxation quadrupoles. J Acoust Soc Am 1983;73(3):10361046.

30. Majumdar A, Hantos Z, Tolnai J, Parameswaran H, Tepper R, Suki B. Estimating the diameter of airways susceptible for collapse using crackle sound. J Appl Physiol 2009;107(5):1504-1512.

31. Malmberg LP, Sovijarvi ARA, Paajanen E, Piirila P, Haahtela T, Katila T. Changes in frequency spectra of breath sounds during histaminic challenge test in adult asthmatics and healthy control subjects. Chest 1994;105(1):122-132.

32. Fiz JA, Jane R, Salvatella D, Izquierdo J, Lores L, Caminal P, et al. Analysis of tracheal sounds during forced exhalation in asthma patients and normal subjects. Chest 1999;116(3):633-638.

33. Murphy RL, Vyshedskiy A, Power-Charnitsky VA, Bana DS, Marinelli PM, Wong-Tse A, Paciej R. Automated lung sound analysis in patients with pneumonia. Respir Care 2004;49(12):1490-1497. 